\title{
Éric Pommier (Dir.), La fenomenología de la donación de Jean-Luc Marion
}

Santiago, Prometeo, 2017

\section{Stéphane Vinolo}

\section{(2) OpenEdition}

\section{Journals}

Édition électronique

URL : http://journals.openedition.org/alter/716

DOI : $10.4000 /$ alter.716

ISSN : 2558-7927

Éditeur :

Association ALTER, Archives Husserl (CNRS-UMR 8547)

Édition imprimée

Date de publication : 31 décembre 2018

Pagination : $267-270$

ISBN : 978-2-9550449-4-0

ISSN : 1249-8947

\section{Référence électronique}

Stéphane Vinolo, «Éric Pommier (Dir.), La fenomenología de la donación de Jean-Luc Marion », Alter [En ligne], 26 | 2018, mis en ligne le 22 janvier 2019, consulté le 24 septembre 2020. URL : http:// journals.openedition.org/alter/716 ; DOI : https://doi.org/10.4000/alter.716 


\section{ÉRIC POMMIER (DIR.), LA FENOMENOLOGÍA DE LA DONACIÓN DE JEAN-LUC MARION, SANTIAGO, PROMETEO, 2017}

Stéphane Vinolo

Les publications en langue espagnole sur la phénoménologie de la donation de Jean-Luc Marion sont rares et donc précieuses. Elles le sont d'autant plus lorsque ce qu'elles apportent à la réflexion va audelà de ce que nous trouvons dans les nombreuses études en langue française consacrées à cet auteur. C'est le cas du livre dirigé par Eric Pommier, fruit d'un colloque organisé à l'Université Catholique de Santiago du Chili. Outre deux articles de Jean-Luc Marion inédits en espagnol («La question de l'inconditionnel » et «Les limites de la phénoménalité »), l'ouvrage présente huit études qui couvrent l'ensemble du spectre sur lequel résonne cette phénoménologie, depuis le problème des fondements phénoménologiques de la donation jusqu'à son impact dans le champ de la théologie, en passant par les rapports de Marion avec Henry, Merleau-Ponty, Husserl, le jeune Heidegger, ou encore Derrida.

La première étude interroge le statut de la chair - troisième figure de la saturation selon la relation - à la lumière de Henry et de Patočka. La chair n'est pas un phénomène saturé comme les autres puisque c'est par la prise de chair que la phénoménalité est possible. Mais, note Pommier, si elle se reçoit elle-même au travers d'une donation, cela suppose une donation originaire eu égard à laquelle elle occupe un statut dérivé, secondaire. Il y a ainsi un appel du monde auquel le sujet - adonné - ne fait que répondre. Néanmoins, d'un autre côté, Marion affirme l'immanence de la chair en tant que condition de l'intentionnalité perceptive. Grâce à Henry qui pose la pleine immanence et la primauté de la chair, et à Patočka qui, au contraire, pose une première donation du monde, et en retravaillant la frontière entre l'auto- 
affection et l'hétéro-affection, l'horizon ou encore le mouvement, Pommier montre que c'est dans la synthèse passive que se trouve la solution au problème de l'immanence de la chair.

Patricio Mena prolonge les analyses sur l'adonné à partir du problème de l'appel, dont il montre combien il est essentiel à la phénoménologie française. Bien qu'ayant mis à mal le sujet transcendantal et sa position de fondement, nombre de phénoménologues ont conservé le transcendantal en le déplaçant du côté de la manifestation du phénomène saturé. Néanmoins cela ne réduit pas le sujet à une passivité face à un phénomène actif ; au contraire, la réception est un type d'activité. C'est alors le statut paradoxal de l'appel qui permet d'expliquer ce point puisque l'appel ne se révèle en tant que tel que dans la réponse qu'y apporte quelqu'un qui s'engage à y répondre, fût-ce négativement. Ainsi, c'est en tant que témoin que l'adonné doit être pensé, témoin qui révèle l'appel par la vocation vécue au long de sa vie.

Luis Flores présente alors une analyse originale des relations entre Husserl et Marion. En partant d'une généalogie de l'objet et de l'objectité chez Husserl, analyse qui l'amène à montrer le long chemin qui amena Husserl à poser des définitions stables, Flores en vient à montrer que contre les philosophes voyeurs de la tradition, Marion propose l'image d'un philosophe flâneur, se laissant aller au gré des phénomènes, suivant leurs manifestations, de la même façon qu'un mineur, pourtant actif, n'en est pas moins obligé de suivre les filons ou les veines des matériaux qu'il souhaite extraire, et donc de se soumettre à la direction qu'ils leur dictent.

La relation avec le premier Heidegger analysée par Francisco de Lara vise à mettre au jour l'enjeu de la donation chez Heidegger et chez Marion. En effet, Marion affirme que le Es gibt heideggérien, qu'il propose de traduire par « cela donne » et non pas par « il y a », tel qu'il apparaît en GA58, nous révèle que la donation est, pour le penseur allemand, l'avenir de la philosophie en même temps qu'elle en marque l'inévitable destin phénoménologique. Or, affirme De Lara, cette interprétation de Marion ne reflète en rien le contexte du débat épistémologique avec les néokantiens dans lequel apparaît le Es gibt. Si Marion s'en était tenu à ce débat, il aurait pu voir que la donation, chez Heidegger, bien qu'importante, ne permet pas de sortir de la nécessaire corrélation (encore que ce ne soit pas avec une conscience) que nous imposent les phénomènes, et donc, qu'elle n'est pas, comme souhaite le voir la phénoménologie de la donation, inconditionnée.

L'étude de Ericson Falabretti présente une grande originalité puisqu'il n'existe pas, en français, d'étude comparée de Marion et de Merleau-Ponty. C'est sur le concept de réduction que s'opère le dialogue. 
Pour Merleau-Ponty, la réduction ouvre l'espace d'une réflexion sur la corrélation entre le mondain et l'activité prépersonnelle du corps-propre. Néanmoins, au fur et à mesure des textes, ce point est qualifié de « mauvaise ambiguïté » qui s'instaure entre l'intériorité et l'extériorité, et porte une tentation de réintroduire un sujet transcendantal premier eu égard à la matière et à la vie. Au contraire, pour Marion, la réduction ne reconduit qu'à la seule donation, et porte donc une «bonne ambiguïté » qui brouille les frontières entre l'intérieur et l'extérieur, le constituant et le constitué, au cœur du pli du donné.

Andrea Potestà cherche, quant à lui, à identifier le lieu précis du débat entre Marion et Derrida. Contrairement à ce que l'on pense traditionnellement, ce lieu n'est pas le don puisqu'au-delà de différences réelles, Derrida et Marion peuvent être rapprochés sur leur opposition à sa conception économique et circulaire, mais la rupture se trouve bien plutôt dans leurs conceptions de la théologie négative. Alors que Derrida, envisageant deux voies (et deux voix), retrouve, au sein de la théologie négative, une affirmation de Dieu qui se joue à un niveau supérieur et en marque l'échec ; Marion, de son côté, pose une troisième voie - celle de la théologie mystique -, qui loin de laisser coexister deux voies, en emprunte une troisième : celle d'un langage non prédicatif qui échappe à toute relation à la présence et à l'absence, et se pose donc sur un autre horizon que celui de l'être. Ce faisant, le dialogue entre Marion et Derrida porte in fine sur la dialectique. Là où Derrida souhaite ne pas dépasser les contradictions mais installer sa pensée en leur cœur, Marion souhaite pousser la sienne jusqu'à l'excès et donc dépasser l'équilibre des voies.

Luis Mariano de la Maza rappelle le rôle prépondérant de l'amour dans la phénoménologie de Marion, et tout particulièrement l'amour en tant qu'il est univoque et ne se distingue plus en eros et agapè. Celuici en effet, affole tous les couples conceptuels qui structurent la pensée métaphysique : activité et passivité, possibilité et effectivité, intimité et étrangèreté, primauté et secondarité. La réduction érotique opère donc une fois de plus un décentrement du sujet et complique la relation entre les amants en posant la nécessité d'un tiers qui apparaît comme témoin de celle-ci depuis son intérieur. C'est là la position de l'enfant, non pas comme entité biologique mais comme événement qui fera don aux amants de la paternité tout autant que de la maternité, qui seront donc reçues, elles aussi, d'ailleurs.

Enfin, Eduardo Silva analyse l'impact de la phénoménologie de la donation dans le champ de la théologie. Après avoir rappelé les enjeux de l'accusation de tournant théologique de la phénoménologie à laquelle a dû faire face Marion, Silva pose, suivant ce faisant les thèses 
de Marion, que la phénoménologie est une des relèves de la métaphysique pour la théologie. On appréciera tout particulièrement les rapprochements opérés entre un certain type de théologie qui abandonne la métaphysique et la théologie de la libération, qui partagent le même horizon. Ce sont les concepts d'événement et d'adonné qui permettent ce nouveau dialogue entre phénoménologie et théologie, en ouvrant un espace dans lequel la pensée de la Révélation échappe à la simplicité de la constitution phénoménologique et se complique dans les boucles de la structure de l'appel.

De tous côtés donc, le livre dirigé par Éric Pommier permettra au lecteur hispanophone de mesurer la profondeur de la phénoménologie de la donation ainsi que les chemins qu'elle ouvre et qui demeurent inexplorés en phénoménologie, éthique, esthétique ou encore en théologie. Mais il permettra aussi au lecteur francophone de s'ouvrir aux problématiques et aux interprétations de Marion qui surgissent depuis un espace géographique dans lequel elles s'avèrent, plus que jamais, fécondes. 